\title{
Reduction in laboratory turnaround time decreases emergency room length of stay
}

This article was published in the following Dove Press journal:

Open Access Emergency Medicine

\section{Nitin Kaushik' \\ Victor S Khangulov ${ }^{2}$ \\ Matthew O'Hara² \\ Ramy Arnaout ${ }^{3,4}$}

'Becton, Dickinson and Company, Franklin Lakes, NJ, USA; ${ }^{2}$ Department of Health Economics and Outcomes Research, Boston Strategic Partners, Inc., Boston, MA, USA; ${ }^{3}$ Department of Pathology, ${ }^{4}$ Division of Biomedical Informatics, Department of Medicine, Beth Israel Deaconess Medical Center, Boston, MA USA

Video abstract

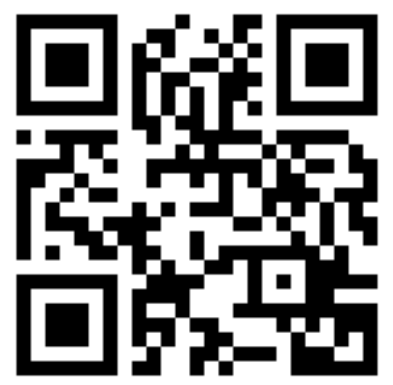

Point your SmartPhone at the code above. If you have a QR code reader the video abstract will appear. Or use: http://youtu.be/7k_tEhGC7-Y
Correspondence: Ramy Arnaout Department of Pathology, Beth Israel Deaconess Medical Center, 330 Brookline Avenue, Boston, MA 02215, USA

Tel + I $617538568 \mid$

Email rarnaout@bidmc.harvard.edu
Objective: Laboratory tests are an important contributor to treatment decisions in the emergency department (ED). Rapid turnaround of laboratory tests can optimize ED throughout by reducing the length of stay (LOS) and improving patient outcomes. Despite evidence supporting the effect of shorter turnaround time (TAT) on LOS and outcomes, there is still a lack of large retrospective studies examining these associations. Here, we evaluated the effect of a reduction in laboratory TAT on ED LOS using retrospective analysis of Electronic Health Records (EHR). Materials and methods: Retrospective analysis of ED encounters from a large, US-based, de-identified EHR database and a separate analysis of ED encounters from the EHR of an ED at a top-tier tertiary care center were performed. Additionally, an efficiency model calculating the cumulative potential LOS time savings and resulting financial opportunity due to laboratory TAT reduction was created, assuming other factors affecting LOS are constant.

Results: Multivariate regression analysis of patients from the multisite study showed that a 1 -minute decrease in laboratory TAT was associated with 0.50 minutes of decrease in LOS. The single-site analysis confirmed our findings from the multisite analysis that a positive correlation between laboratory TAT and ED LOS exists in the ED population as a whole, as well as across different patient acuity levels. In addition, based on the calculations from the efficiency model, for a 5-, 10- and 15-minute TAT reduction, the single-site ED can potentially admit a total of 127, 256 and 386 additional patients, respectively, annually.

Conclusion: A positive correlation between laboratory TAT and ED LOS was observed in a broad patient population and across distinct acuity levels.

Keywords: laboratory testing, turnaround time, emergency department, length of stay, patient acuity, retrospective analysis, data science

\section{Introduction}

With 140 million patients presenting through emergency departments (EDs) annually, overcrowding and long wait times are a major concern for hospitals across the USA. ${ }^{1}$ From 2003 through 2009, the mean wait time to see a provider (physician, physician's assistant or nurse practitioner) in US EDs increased by $25 \%$ from 47 to 58 minutes. ${ }^{2,3}$ Long ED stays can have profound consequences, such as avoidable medical errors, negative impact on patient outcomes and higher mortality. ${ }^{4-7}$ ED length of stay (LOS) directly impacts rates of inpatient admission and hospital LOS and can add to the overall burden on the hospital system. ${ }^{8-10}$ A previous study analyzing the 2008 National Hospital Ambulatory Medical Care Survey reported a median LOS of 269 minutes for safety-net EDs and 281 minutes for non-safety-net EDs. ${ }^{11}$ For many patients, the ED is the first point of access to the hospital; thus, LOS in the ED is considered an 
important indicator of quality of care for the hospital as a whole. ${ }^{12,13}$ In 2008 , a study analyzing 1,500 hospitalizations reported that long waits and delayed treatment in the ED negatively impacted patient satisfaction. ${ }^{14}$

Results of laboratory tests ordered in the ED are an important factor in patient-management decisions. Appropriate triage and discharge of patients is impacted by the timely return of laboratory test results, ${ }^{4,15}$ making turnaround time (TAT) of laboratory tests a key contributor to ED workflow. ${ }^{15}$ The ability to provide fast turnaround of samples is considered an essential performance metric of the laboratory ${ }^{15,16}$ and appears to improve the operational efficiency of the ED. ${ }^{17,18}$ For example, rapid troponin testing helps to confirm or rule out myocardial infarction in ED patients ${ }^{19}$ and can significantly impact patient mortality. In addition, a 2015 study reported that quick turnaround of test results in patients with acute respiratory tract illness admitted to the ED resulted in a faster therapeutic intervention and better patient outcomes. ${ }^{20}$ Thus, as hospitals make efforts to improve ED performance, it is important to consider that improving factors outside of the department, such as laboratory TAT, may improve the operational efficiency of the ED. We sought to address the possibility that reduction in laboratory TAT can shorten ED LOS by testing the hypothesis that TAT and ED LOS are positively correlated.

\section{Materials and methods}

We conducted two studies. The first involved a multisite retrospective analysis of Electronics Health Records (EHR) data from 486 hospitals across the USA. The second was an EHR analysis of Beth Israel Deaconess Medical Center's (BIDMC) ED, which incorporated patient segmentation by four levels of acuity. Based on our findings from the second analysis, we also created a Microsoft Excel-based tool to understand the impact of efficiency on patient LOS and throughput.

\section{Multisite EHR analysis of laboratory TAT and ED LOS \\ Study design and data source}

We conducted a retrospective analysis using Cerner Health Facts $^{\circledR}$, an electronic database of aggregated, de-identified clinical and administrative data from 486 US hospitals and health systems. Institution review board approval for the multisite study was not required since all the data obtained from the Cerner database were de-identified, making our study exempt.

\section{Patient population}

We considered a "treat-and-release" population, that is, patients not admitted to inpatient services directly from the
ED who spent $<7$ hours in the ED. This choice was based on the 2011 National Hospital Ambulatory Medical Care Survey data wherein $>86 \%$ of ED visits were $<7$ hours in duration, and $73 \%$ of all 2012 ED visits in the database were treat-and-release with discharge home, ${ }^{21}$ making our selection representative of the general ED population.

\section{Inclusion and exclusion criteria}

Patients who had an ED admission between January 1, 2012 and December 31, 2012; were $\geq 18$ years old; had an ED LOS $<7$ hours ( $0-419$ minutes) and $\geq 1$ laboratory test performed during ED admission; and were discharged home to care of family/friend(s), a paid caregiver or had a regular discharge with follow-up were included. Patients were excluded if they had a lab TAT $<5$ minutes (assumed to be point-of-care tests and, therefore, not relevant to this study); missing gender or laboratory test data; laboratory test data with one or more missing timestamps; test results returned post-discharge in spite of having being ordered within the defined TAT start time (which is 30 minutes from the first test ordered).

The final cohort represented $50.3 \%$ of adult ED patients in the database with $\geq 1$ laboratory test ordered and valid laboratory data. The effect of each of the main filters is shown in Table S1.

\section{Definitions}

TAT start time was defined as the later of laboratory order/ draw time for the first test (blood and/or urine) ordered; end time was defined as the time of completion of the last test ordered within 30 minutes of the first test (Figure 1). LOS was defined as the time elapsed between ED admission and discharge, as recorded in the database.

\section{Statistical analysis}

Relationships between TAT and LOS were examined via a two-level linear mixed-effect model with truncation (at 419 minutes) using hospitals as the second level to account for institution-specific effects. Variables used in the analysis were age, gender, race, severity of illness, admission source and type, payer type, hospital type and bed size, hospital setting, census region, day of test and overall test count. Confounders were identified by manual substitution of individual factors (e.g., demographics, payer type, severity of illness, hospital characteristics and test volume) into the regression model. Severity of illness was measured by the Rapid Emergency Medicine Score. For regression analyses, strength of the relationship between the TAT and LOS was assessed based on the statistical significance of the slope 

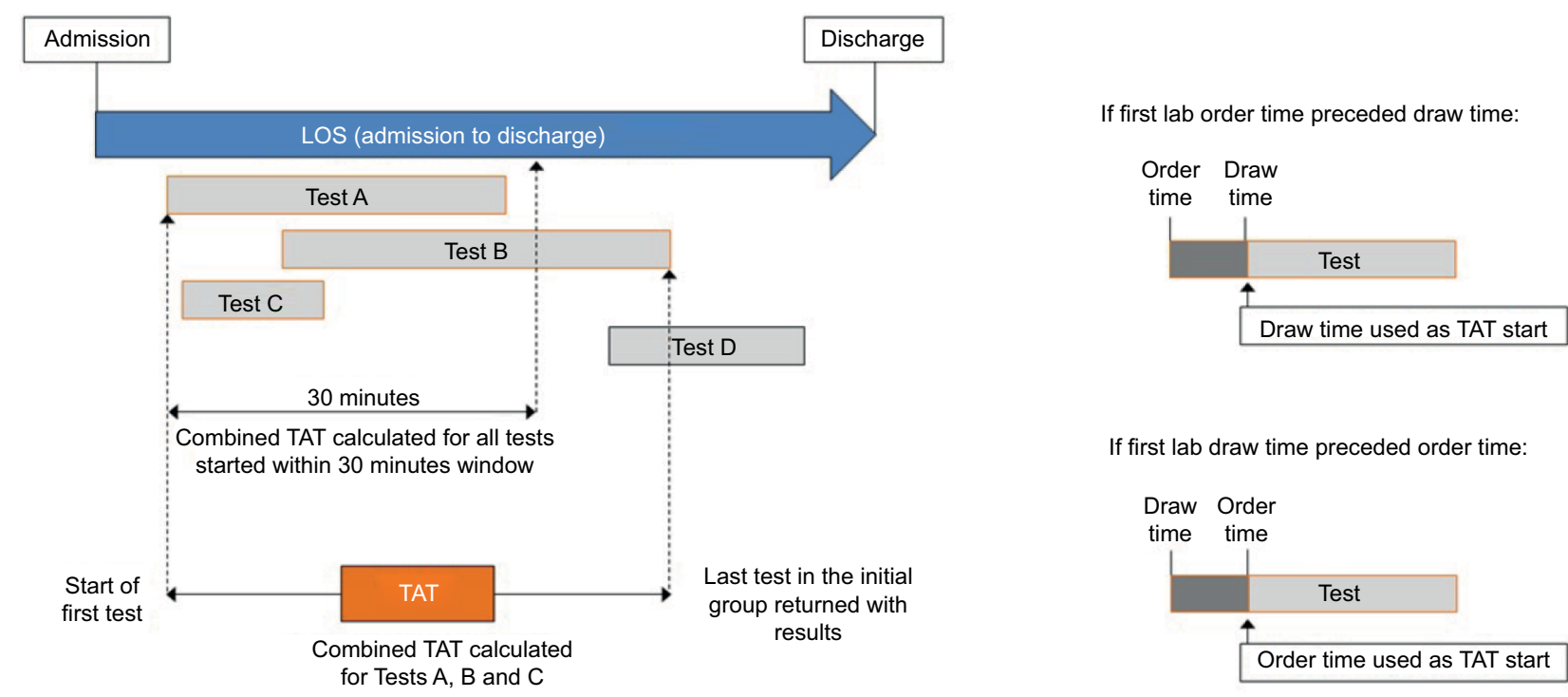

Figure I Definition of laboratory TAT.

Notes: TAT start time was defined as the latest of lab order/draw time for the first test ordered; end time was defined as the time of completion of the last test ordered within 30 minutes of the first test. Test A-D represent multiple tests used to determine the Lab TAT within the defined time period.

Abbreviations: LOS, length of stay; TAT, turnaround time.

coefficient differing from zero $(P$-value of $<0.05$ denoted statistical significance).

\section{Single-site (BIDMC EHR) analysis of laboratory TAT and ED LOS Study design and data source}

A second retrospective analysis was conducted using hospital records from the BIDMC ED. These records contained 52,080 visits on 36,570 unique patients from 2012 to 2015. The EHR data included timestamped information for admissions, discharges, laboratory orders and specimen collections. For this study, we obtained institution review board approval from the BIDMC expedited under Category 5 . The primary objective of this single-site study was to extend our findings from the multisite study by studying the differential impact of laboratory TAT on ED LOS based on patient acuity. Based on the results of this analysis, an efficiency model was created to demonstrate the time savings and benefits of BIDMC.

\section{Patient population}

Sequential inclusion and exclusion criteria (Table S2) were applied to reflect treat-and-release patients admitted to the BIDMC ED. The total number of patient visits to the ED was tabulated for a consecutive 12-month period from 2012 to 2015 (our anonymization process removed the specific year to protect patient privacy).

Acuities ranging from 1 through 4 were assigned at the time of triage by the physician, based on the severity of the condition presented, with 1 being the lowest severity and 4 being the highest (Table S3).

\section{Inclusion and exclusion criteria}

Subjects who were $\geq 18$ years old, had at least one blood or urine test ordered in the laboratory during the visit, had an assigned gender (male or female), an LOS $<7$ hours, were sent home after treatment and had principal complaint listed were included (Table S3). Subjects were excluded if encounters had missing, incomplete or uninterpretable laboratory timestamps (e.g., laboratory drawn dates and times). Tests with results not available prior to the discharge time were excluded from the TAT calculation as the results from these tests did not impact patient's LOS; canceled tests were also excluded.

\section{Definitions}

TAT definition was similar to the multisite study (the duration of time between the sample collection and the result; Figure 1) and calculated for tests run at BIDMC (i.e., not send-out tests). LOS was defined as the time elapsed between ED admission and discharge as recorded in the BIDMC EHR system.

\section{Statistical analysis}

The association between TAT and LOS was examined via truncated regression (PROC QLIM, SAS 9.4) to address the artificial boundary imposed on the data by LOS $<7$ hours. Each acuity segment was analyzed using an independent 
regression model to calculate the coefficients for TAT and to estimate the change in LOS per 1-minute TAT. The variables included in the analysis were TAT, gender, age, source of patient arrival, time of day and day of week of the visit, calendar quarter, principal complaint and discharge ICD9International Classification of Diseases, Ninth Revision code (Table S3). The relationship between TAT and LOS was assessed based on the estimated coefficient of TAT and its statistical significance (as mentioned earlier, using the conventional significance cutoff of $P=0.05$ ).

\section{Efficiency model generation from single-site EHR analysis}

We used the four correlation coefficients (one per acuity) between laboratory TAT and ED LOS to calculate a hypothetical annual LOS reduction and additional patient throughput, making a simplifying assumption that the ED is operating at capacity.

\section{Results}

The initially identified patient population for the multisite study had 4,483,169 ED visits, and following application of inclusion and exclusion criteria, a final cohort of 463,712 visits was obtained for further analysis (Table S1). Of the variables included in the analysis, race, Rapid Emergency Medicine Score, hospital bed size, hospital type teaching, census region and overall test counts were identified as confounders and were adjusted for in the model. A positive correlation between TAT and LOS was observed, particularly for patients admitted to the ED and subsequently discharged home following treatment. Based on these findings, a second, a more refined, single-site study that incorporated patient segmentation by four levels of acuity was conducted to further test our initial hypothesis. This second study had a final cohort of 11,247 patients. An efficiency model for each acuity, calculating the cumulative potential LOS time savings and opportunity to accept additional patients due to laboratory TAT reduction, was created, assuming that other factors affecting LOS were unchanged.

\section{Association between LOS and TAT}

For the multisite EHR analysis, there was a positive association between LOS and TAT (Figure 2A). We also observed that median LOS increased with 15-minute laboratory TAT intervals and that shorter TATs were associated with wider LOS ranges (Figure 2B). To confirm these observations while accounting for potential confounders, we used regression to analyze TAT as a continuous independent variable. We calculated that a 1-minute decrease in TAT was associated with a 0.5 -minute decrease in LOS, holding all other variables constant (Table 1).

\section{Calculated coefficients of covariates from each acuity significant to LOS}

Multiple variables corresponding to each patient visit and acuity were analyzed. The variables significant to the_LOS $(P<0.05)$ were accepted for further multivariate regression analysis. Correlation coefficients for TAT were calculated using a multivariate regression with LOS as a dependent variable (Table 1). For acuities 1, 2, 3 and 4, every 1 -minute increase in TAT resulted in 0.37-, 0.52-, 0.57- and 0.90 -minute increases in LOS, respectively. The weighted
A

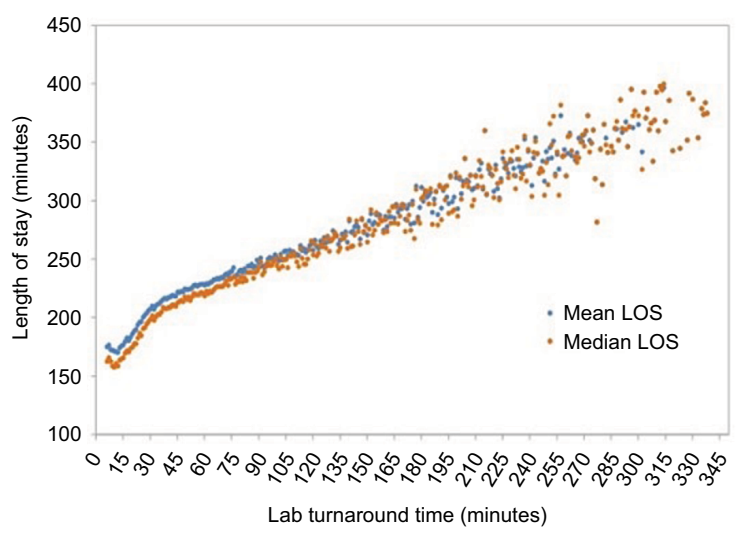

B

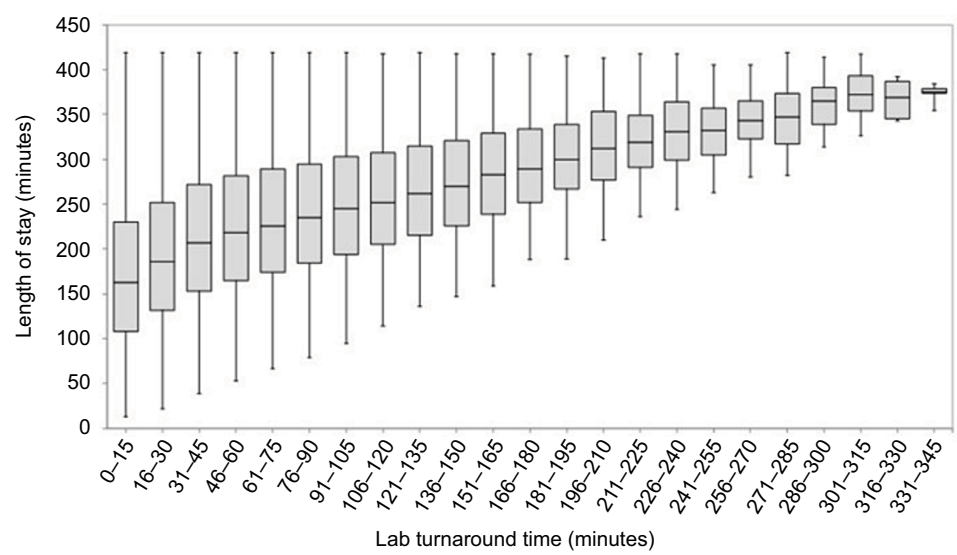

Figure 2 ED LOS correlates with laboratory test TAT.

Notes: (A) Plotting mean and median ED LOS as a function of laboratory test TAT reveals positive correlations with TAT. (B) Box and whisker plot indicates that median LOS increases with each 15 minutes laboratory TAT interval, and that shorter TATs are associated with wider LOS ranges. Horizontal line = median LOS; box = LOS values within upper and lower quartiles; whiskers $=$ LOS range.

Abbreviations: ED, emergency department; LOS, length of stay; TAT, turnaround time. 
average, a 0.55 -minute shorter LOS per minute saved in TAT, was similar to what we observed for the multisite study (0.50-minute shorter LOS per minute saved in TAT). Higher acuities had a stronger positive association between TAT and LOS.

\section{Total annual benefit of LOS saving for a 5-, 10- and I5-minute reduction in TAT}

Assuming 5-, 10- or 15-minute per-patient reductions in TAT, we calculated the total annual LOS saving for each acuity segment (Table 2). With higher reduction in TAT, there were greater reductions in LOS across all acuities. For a 5-, 10or 15-minute reduction in TAT, we calculated total annual LOS savings to be 519, 1,038 and 1,557 hours, respectively. Additionally, the largest per-visit gain was observed for the highest-acuity patients. We also calculated the annual increase in the hospital's ability to accept additional patients due to TAT reduction. Across all acuities, for a 5-, 10- and 15-minute TAT reduction, the BIDMC ED could potentially admit 127, 256 and 388 additional patients, respectively, per year.

\section{Discussion}

Our retrospective analysis of real-world patient data from 486 US hospitals indicated a 0.50 minute decrease in ED

Table I Decrease in LOS as a function of TAT, by patient acuity

\begin{tabular}{lll}
\hline Acuity level & $\begin{array}{l}\text { Decrease in LOS, in minutes, } \\
\text { for each minute of TAT }\end{array}$ & $P$-value \\
\hline 1 & 0.37 & $<0.000 \mathrm{I}$ \\
2 & 0.52 & $<0.000 \mathrm{I}$ \\
3 & 0.57 & $<0.000 \mathrm{I}$ \\
4 & 0.91 & $<0.000 \mathrm{I}$ \\
Weighted average & 0.55 & \\
\hline
\end{tabular}

Note: Weighted average was calculated using the numbers of visits by acuity in Table 2.

Abbreviations: LOS, length of stay; TAT, turnaround time.
LOS with every 1 -minute decrease in laboratory TAT. We confirmed these results for data from a single tertiary medical center (BIDMC), where patients were segmented based on acuity; we found that a 1-minute decrease in laboratory TAT for patients across acuities $1-4$ resulted in $0.37-0.91$ minute reductions in ED LOS, respectively, with the greatest gains for the highest-acuity patients. Together, these results suggest that they generalize to ED populations nationwide.

To understand how this TAT-LOS relationship might impact the ED on an annual timescale, we created an efficiency model. For a hospital with the size and distribution of acuities of BIDMC, a tertiary health care center, we calculated a savings of 519 hours in total LOS for a reduction of as little as 5 minutes in TAT, with proportionately greater savings for greater reductions in TAT (Table 2). This trend was consistent across all acuities; however, patients in acuity 3, with the highest overall patient volume (two-thirds of total visits), had the highest cumulative reduction in LOS. In addition, by calculating the annual LOS savings for each acuity, we were able to estimate the number of additional patients that could be admitted to a site like BIDMC due to the reduction of laboratory TAT. For 5-, 10- and 15-minute total reductions in average TAT per patient, BIDMC ED could potentially accept $\sim 126,256$ and 388 additional patients, respectively, which is an overall increase of $1 \%-3 \%$ (Table 2 ). The ability to accept and provide treatment to additional patients would directly correlate with improving patient flow in the ED. Such savings offer a significant opportunity for service improvement and potential profit sharing for the clinical laboratories, with similar opportunities and knock-on synergies throughout the hospital.

Additionally, a reduction in the ED LOS can have several other positive impacts on the patient and hospital: wait time for new patients awaiting an empty bed, number of patients who left without being seen and ambulance diversion rates may be reduced, with attendant potential improvements in patient satisfaction and outcomes. A reduction in LOS can

Table 2 TAT, LOS, estimated LOS reduction and estimated additional admissions by patient acuity level

\begin{tabular}{|c|c|c|c|c|c|c|c|c|c|c|}
\hline \multirow[t]{2}{*}{$\begin{array}{l}\text { Acuity } \\
\text { level }\end{array}$} & \multirow[t]{2}{*}{ Visits } & \multirow[t]{2}{*}{$\begin{array}{l}\text { Median } \\
\text { TAT, hours }\end{array}$} & \multirow{2}{*}{$\begin{array}{l}\text { Mean } \\
\text { LOS, } \\
\text { hours }\end{array}$} & \multirow{2}{*}{$\begin{array}{l}\text { Median } \\
\text { LOS, } \\
\text { hours }\end{array}$} & \multicolumn{3}{|c|}{$\begin{array}{l}\text { Annual LOS reduction, in hours, from } \\
\text { a mean TAT reduction of }\end{array}$} & \multicolumn{3}{|c|}{$\begin{array}{l}\text { Annual additional admissions from a } \\
\text { mean TAT reduction of }\end{array}$} \\
\hline & & & & & 5 minutes & 10 minutes & 15 minutes & 5 minutes & 10 minutes & 15 minutes \\
\hline I & 631 & 0.9 & 4.3 & 4.4 & 20 & 39 & 59 & 5 (I\%) & 9 (1\%) & $14(2 \%)$ \\
\hline 2 & 2,662 & 0.9 & 4.4 & 4.4 & 114 & 229 & 343 & $26(1 \%)$ & $53(2 \%)$ & $80(3 \%)$ \\
\hline 3 & 7,575 & 0.9 & 4.1 & 4.1 & 356 & 713 & 1,069 & 87 (1\%) & 176 (2\%) & $268(4 \%)$ \\
\hline 4 & 379 & 0.7 & 3.4 & 3.2 & 29 & 57 & 86 & $9(2 \%)$ & $18(5 \%)$ & 27 (7\%) \\
\hline Total & I I,247 & 0.9 & 4.2 & 4.1 & 519 & 1,038 & $\mathrm{I}, 557$ & 127 (।\%) & $256(2 \%)$ & $389(3 \%)$ \\
\hline
\end{tabular}

Note: Numbers in parentheses are percentages of the number of visits by acuity.

Abbreviations: LOS, length of stay; TAT, turnaround time. 
also lead to better fixed cost distribution (efficient staff utilization) and, consequently, an improvement in institution's profitability. There are several ways through which laboratories can potentially improve laboratory TAT, including at pre-analytical (e.g., specimen handling), analytical (moving to a sample type such as plasma which does not require clotting, reducing centrifugation time for samples, optimizing laboratory workflow by eliminating unnecessary steps) and post-analytical (results reporting) steps. Not to be ignored are potential process speedups in test selection and results interpretation (the so-called pre-pre- and post-post-analytical steps), which are currently major contributors to diagnostic and laboratory-related medical error and are the frontier in laboratory quality. ${ }^{22}$ Our work points to the specific quantitative benefits to the ED of such improvements, and thus can inform resource allocation decisions within and between the laboratory and the ED.

To fully understand the implications of laboratory TAT and hospital LOS reductions, future studies investigating the impact of laboratory TAT on factors such as wait time, hospital admittance (beyond treat-and-release patient group), left without being seen rates and ED throughput are warranted, as well as segmentation to study the contributions of specific laboratory tests or panels. We also observed that certain patient conditions, characteristics and diagnosis groups had a more significant impact on LOS (Table S3); these were adjusted for in our models. Studies examining specific patient groups and tests would likewise provide additional insights.

It is important to note certain limitations of this study. For our multisite study, we were not able to capture local practice patterns (e.g., triage process, testing protocols, patient to clinician ratio and so on), and although we incorporated the hospital variable in the two-stage model, there is no way for us to obtain tailored guidelines for specific institutions. Also, we note that laboratory TAT is only one of the factors impacting the ED LOS of the patient, and there are several other variables that could prolong the ED LOS. Other factors such as radiology (e.g., X-rays and computed tomography scans) and availability of specialists can affect the patient flow and decision-making process, and the impact of these factors was not analyzed in our study. Additionally, time of decision making after receiving a test result can also impact overall LOS. A recent study reported a correlation between physician experience and rapidity of decision making, so future models may benefit from the inclusion of variables such as time in practice. ${ }^{23}$

\section{Conclusion}

Hospitals across the USA are continuing to experience pressure to treat more patients with fewer EDs and fewer hospital beds. The ED has more complex workflows compared to other hospital departments, and crowding in the ED based on the severity of the disease can affect patients differently. ${ }^{2,24,25}$ Here we find that even very modest decreases in the laboratory TAT can have appreciable impact on ED LOS. Additionally, our efficiency model suggests that reduction of TAT will allow the ED to accept more patients, thus highlighting the importance and benefit of developing processes/technologies with the potential to drive further efficiencies and reduce laboratory TAT.

\section{Acknowledgments}

The authors acknowledge Hussain Badani, $\mathrm{PhD}$, and David K Hayashida, MSc, of Boston Strategic Partners, Inc. for editorial assistance. This study was funded by Becton, Dickinson and Company ([BD]; Franklin Lakes, NJ, USA).

\section{Author contributions}

All authors contributed toward data analysis, drafting and revising the paper and agree to be accountable for all aspects of the work.

\section{Disclosure}

$\mathrm{MOH}$ and VSK were paid by BD to perform the analysis and provide editorial support on the manuscript. RA received an educational grant from BD to conduct the single-site study and provide editorial support for the manuscript. The authors report no other conflicts of interest in this work.

\section{References}

1. Wiler JL, Welch S, Pines J, Schuur J, Jouriles N, Stone-Griffith S. Emergency department performance measures updates: proceedings of the 2014 emergency department benchmarking alliance consensus summit. Acad Emerg Med. 2015;22(5):542-553.

2. Hing E, Bhuiya F. Wait time for treatment in hospital emergency departments: 2009. 2012. Available from: https://www.cdc.gov/nchs/ data/databriefs/db102.htm. Accessed January 26, 2017.

3. TrendWatch Chartbook 2013. 2013. Available from: http://www.aha. org/research/reports/tw/chartbook/2013/13chartbook-full.pdf. Accessed January 26, 2017.

4. Li L, Georgiou A, Vecellio E, et al. The effect of laboratory testing on emergency department length of stay: a Multihospital Longitudinal Study applying a cross-classified random-effect modeling approach. Acad Emerg Med. 2015;22(1):38-46.

5. Epstein SK, Huckins DS, Liu SW, et al. Emergency department crowding and risk of preventable medical errors. Intern Emerg Med. 2012;7(2):173-180

6. Richardson DB. Increase in patient mortality at 10 days associated with emergency department overcrowding. Med J Aust. 2006;184(5):213-216. 
7. Horwitz LI, Green J, Bradley EH. US emergency department performance on wait time and length of visit. Ann Emerg Med. 2010;55(2):133-141.

8. Carrier E, Khaldun J, Hsia RY. Association between emergency department length of stay and rates of admission to inpatient and observation services. JAMA Intern Med. 2014;174(11):1843-1846.

9. Peck JS, Benneyan JC, Nightingale DJ, Gaehde SA. Predicting emergency department inpatient admissions to improve same-day patient flow. Acad Emerg Med. 2012;19(9):E1045-E1054.

10. Huang Q, Thind A, Dreyer JF, Zaric GS. The impact of delays to admission from the emergency department on inpatient outcomes. BMC Emerg Med. 2010;10:16.

11. Fee C, Burstin H, Maselli JH, Hsia RY. Association of emergency department length of stay with safety-net status. JAMA. 2012;307(5):476-482.

12. Kaboli PJ, Go JT, Hockenberry J, et al. Associations between reduced hospital length of stay and 30-day readmission rate and mortality: 14-year experience in 129 Veterans Affairs Hospitals. Ann Intern Med. 2012;157(12):837-845.

13. Mitral D, Erdal E, Khangulov V, Tuttle R. Association between laboratory test turnaround time and emergency department length of stay: a retrospective electronic health record database study. Poster presented at the American Academy for Clinical Chemistry Annual Meeting \& Clinical Lab Expo; July 26-30; 2015; Atlanta, GA.

14. Pines JM, Iyer S, Disbot M, Hollander JE, Shofer FS, Datner EM. The effect of emergency department crowding on patient satisfaction for admitted patients. Acad Emerg Med. 2008;15(9):825-831.

15. Hawkins RC. Laboratory turnaround time. Clin Biochem Rev. 2007;28(4):179-194.

16. Singer AJ, Viccellio P, Thode HC, Bock JL, Henry MC. Introduction of a stat laboratory reduces emergency department length of stay. Acad Emerg Med. 2008;15(4):324-328.
17. Bashkin O, Caspi S, Haligoa R, Mizrahi S, Stalnikowicz R. Organizational factors affecting length of stay in the emergency department: initial observational study. Isr J Health Policy Res. 2015;4:38.

18. Erenler AK, Akbulut S, Guzel M, et al. Reasons for overcrowding in the emergency department: experiences and suggestions of an education and research hospital. Turk J Emerg Med. 2016;14(2):59-63.

19. Cullen L, Parsonage W, Than M, et al. Myocardial infarction: rapid ruling out in the emergency room. Lancet. 2015;386(10012): 2449-2450.

20. Rogers BB, Shankar P, Jerris RC, et al. Impact of a rapid respiratory panel test on patient outcomes. Arch Pathol Lab Med. 2015;139(5) 636-641.

21. Centers for Disease Control and Prevention. National Hospital Ambulatory Medical Care Survey: 2011 Emergency Department; Summary Tables. Available from: https:/www.cdc.gov/nchs/data/ahcd/ nhamcs_emergency/2011_ed_web_tables.pdf. Accessed February 15, 2018.

22. Zhi M, Ding EL, Theisen-Toupal J, Whelan J, Arnaout R. The landscape of inappropriate laboratory testing: a 15-year meta-analysis. PLoS One. 2013;8(11):e78962.

23. Asha SE, Chan ACF, Walter E, et al. Impact from point-of-care devices on emergency department patient processing times compared with central laboratory testing of blood samples: a randomised controlled trial and cost-effectiveness analysis. Emerg Med J. 2014;31(9): 714-719.

24. Bernstein SL, Aronsky D, Duseja R, et al; Society for Academic Emergency Medicine, Emergency Department Crowding Task Force. The effect of emergency department crowding on clinically oriented outcomes. Acad Emerg Med. 2009;16(1):1-10.

25. McCarthy ML, Aronsky D, Jones ID, et al. The emergency department occupancy rate: a simple measure of emergency department crowding? Ann Emerg Med. 2008;51(1):15-24.e2. 


\section{Supplementary materials}

Table SI Inclusion and exclusion criteria to narrow the cohort for multisite study

\begin{tabular}{ll}
\hline Criteria & Hospital visits \\
\hline ED visits & $4,483,169$ \\
ED visits from hospitals reporting laboratories to Cerner & $4,027,564$ \\
ED visits with at least one laboratory test & $1,096,724$ \\
LOS $\geq 0$ minutes & $1,096,167$ \\
Age $\geq 18$ years & 945,342 \\
Assigned gender (male or female only) & 944,668 \\
Exclude patients with all cancelled tests & 944,668 \\
Laboratory drawn and complete date-times are populated & 944,668 \\
Laboratory is drawn within LOS & 922,546 \\
LOS $<7$ hours & 642,322 \\
"Treat-and-release" discharge dispositions & 515,473 \\
Exclude patients where laboratory results came after discharge & 483,429 \\
Exclude patients with TAT $\leq 5$ minutes & 463,712 \\
\hline
\end{tabular}

Abbreviations: ED, emergency department; LOS, length of stay; TAT, turnaround time.

Table S2 Inclusion and exclusion criteria to narrow the cohort for single-site study

\begin{tabular}{ll}
\hline Criteria & Hospital visits \\
\hline Data as delivered & 52,080 \\
Age $\geq 18$ years & 51,892 \\
With at least one blood-based laboratory & 30,817 \\
Assigned gender (male or female only) & 30,817 \\
"Treat-and-release", that is, patient departed hospital & 16,425 \\
Non-missing principal complaint & 16,421 \\
LOS $<7$ hours & $1 \mathrm{I}, 247$ \\
Available visits & $\mathrm{II}, 247$ \\
\hline
\end{tabular}

Abbreviation: LOS, length of stay.

Table S3 Patient demographics and distribution by acuity for the single-site study

\begin{tabular}{|c|c|c|c|c|c|c|}
\hline Patient demographics & Acuity level & $\mathbf{I}$ & 2 & 3 & 4 & Total \\
\hline & $\mathbf{n}$ & 631 & 2,662 & 7,575 & 379 & I I,247 \\
\hline Chief complaint & Other pain loci & 3.0 & 6.8 & 14.1 & 23.5 & 12.1 \\
\hline \multirow[t]{9}{*}{ (top I0), \% } & Abdominal pain & 2.2 & 5.4 & 15.3 & 0.8 & 11.8 \\
\hline & Nausea/vomiting/diarrhea & 3.0 & 3.6 & 10.2 & 0.8 & 7.9 \\
\hline & Chest pain & 2.4 & 13.2 & 4.8 & 0.3 & 6.5 \\
\hline & Headache & 5.7 & 5.1 & 4.8 & 2.4 & 4.8 \\
\hline & Back pain & 2.9 & 2.3 & 4.5 & 16.1 & 4.3 \\
\hline & Dizziness & 7.0 & 4.5 & 3.0 & 0.8 & 3.5 \\
\hline & Status post-fall & 3.0 & 5.4 & 2.9 & 3.2 & 3.5 \\
\hline & Dyspnea & 4.4 & 6.2 & 2.3 & 0.0 & 3.3 \\
\hline & Fever & 1.9 & 3.0 & 3.0 & 0.5 & 2.8 \\
\hline \multirow[t]{2}{*}{ Gender, \% } & Female & 54.7 & 57.1 & 65.3 & 71.5 & 63.0 \\
\hline & Male & 45.3 & 42.9 & 34.7 & 28.5 & 37.0 \\
\hline \multirow[t]{7}{*}{ Age, years, \% } & $18-19$ & 4.0 & 3.9 & 4.0 & 3.7 & 4.0 \\
\hline & $20-29$ & 21.9 & 16.6 & 23.2 & 28.5 & 21.7 \\
\hline & $30-39$ & 17.6 & 14.4 & 17.3 & 20.8 & 16.7 \\
\hline & $40-49$ & 12.4 & 14.2 & 15.2 & 13.7 & 14.8 \\
\hline & $50-59$ & 17.9 & 17.9 & 16.1 & 14.0 & 16.6 \\
\hline & $60-64$ & 8.2 & 8.0 & 6.5 & 4.7 & 6.9 \\
\hline & $\geq 65$ & 18.1 & 25.1 & 17.7 & 14.5 & 19.4 \\
\hline
\end{tabular}


Table S3 (Continued)

\begin{tabular}{|c|c|c|c|c|c|c|}
\hline Patient demographics & Acuity level & $\mathbf{I}$ & 2 & 3 & 4 & Total \\
\hline & $\mathbf{n}$ & 631 & 2,662 & 7,575 & 379 & 11,247 \\
\hline \multirow[t]{2}{*}{ Arrival day, \% } & Monday-Friday & 74.5 & 76.7 & 71.0 & 69.7 & 72.5 \\
\hline & Weekend & 25.5 & 23.3 & 29.0 & 30.3 & 27.5 \\
\hline \multirow[t]{2}{*}{ Arrival time, \% } & Day shift $(9 \mathrm{am}-5 \mathrm{pm})$ & 47.5 & 46.0 & 44.9 & 45.4 & 45.3 \\
\hline & Night shift & 52.5 & 54.0 & 55.1 & 54.6 & 54.7 \\
\hline \multirow[t]{2}{*}{ Mode of arrival, \% } & Ambulance & 43.9 & 33.3 & 19.3 & 10.6 & 23.7 \\
\hline & Non-ambulance & 56.1 & 66.7 & 80.7 & 89.4 & 76.3 \\
\hline Discharge diagnosis & Signs/symptoms & 10.1 & 14.8 & 25.3 & 5.5 & 21.3 \\
\hline \multirow[t]{9}{*}{ category (top I0), \% } & Nervous system and sense & 28.2 & 14.4 & 10.0 & 5.8 & 11.9 \\
\hline & Circulatory & 13.5 & 17.5 & 7.8 & 3.2 & 10.3 \\
\hline & Genitourinary & 2.1 & 5.3 & 10.8 & 16.6 & 9.2 \\
\hline & Injury/poisoning & 16.3 & 9.2 & 7.1 & 17.7 & 8.5 \\
\hline & Respiratory & 7.3 & 8.0 & 8.8 & 5.8 & 8.4 \\
\hline & Musculoskeletal/connective tissue & 6.7 & 4.9 & 8.6 & 25.6 & 8.2 \\
\hline & Digestive & 1.0 & 4.8 & 7.3 & 1.6 & 6.2 \\
\hline & Mental illness & 3.5 & 8.0 & 2.5 & 0.8 & 3.8 \\
\hline & Pregnancy/childbirth/puerperium & 1.7 & 2.7 & 3.2 & 1.6 & 2.9 \\
\hline
\end{tabular}

Open Access Emergency Medicine

\section{Publish your work in this journal}

The Open Access Emergency Medicine is an international, peerreviewed, open access journal publishing original research, reports, editorials, reviews and commentaries on all aspects of emergency medicine. The manuscript management system is completely online and includes a very quick and fair peer-review system, which is all

\section{Dovepress}

easy to use. Visit http://www.dovepress.com/testimonials.php to read real quotes from published authors. 